\title{
Hospitalization rate and outcomes in patients with left ventricular dysfunction receiving hemodialysis
}

This article was published in the following Dove Press journal: International Journal of General Medicine

\author{
Marwan A Albeshri' \\ Mohammed S Alsallum' \\ Sulafa Sindi ${ }^{1}$ \\ Mohammed Kadi \\ Abdullah Albishri ${ }^{2}$ \\ Hanadi Alhozali ${ }^{3}$ \\ Kamal Alghalayini ${ }^{3}$ \\ 'College of Medicine, King Abdulaziz \\ University, Jeddah, Saudi Arabia; \\ ${ }^{2}$ College of Medicine, King Abdulaziz \\ University-Rabigh Branch, Rabigh, \\ Saudi Arabia; ${ }^{3}$ Department of Internal \\ Medicine, King Abdulaziz University \\ Hospital, Jeddah, Saudi Arabia
}

Introduction: Left ventricular dysfunction (LVD) is characterized as left ventricular ejection fraction (EF) below half of the systolic capacity of the left ventricle. Patients on hemodialysis have higher risk of developing LVD than the general population. Our aim was to assess hospitalization rate and outcomes in hemodialysis patients with LVD.

Patients and methods: All patients $\geq 18$ years old, who started hemodialysis therapy at King Abdulaziz University Hospital between January 2011 and December 2011, were identified using medical records of hemodialysis unit. Patients were then divided into three groups, according to their EF results prior to the initiation of hemodialysis, as patients with $\mathrm{EF}<40 \%$, EF between $40 \%$ and $49 \%$, and $\mathrm{EF} \geq 50 \%$. Patients were then followed for 5 years by reviewing their hospital records to assess their outcomes, hospital admissions, and length of hospital stay.

Results: Analysis included 333 patients. Patients with EF $<40 \%$ were 40, 36 patients with EF $40 \%-49 \%$, and 257 patients had an EF $>50 \%$. Patients with EF $<50 \%$ were significantly older than patients with $\mathrm{EF}>50 \%(P=0.002)$. Diabetes mellitus and hypertension were more prevalent in patients with $\mathrm{EF}<40 \%$ and $\mathrm{EF} 40 \%-49 \%$ when compared with patients with $\mathrm{EF}>50 \%$ $(P<0.001, P=0.002)$. The average length of stay between the three groups was significantly different $(P=0.007)$. Intensive care unit admissions were significantly different when comparing the three groups $(P=0.013)$ and was found to be an independent risk factor for mortality in our patients. Half of the patients with $\mathrm{EF}<40 \%$ and $44 \%$ of patients with $\mathrm{EF}$ of $40 \%-49 \%$ died compared with only $27 \%$ of patients with EF $>50 \%(P=0.002)$. However, Kaplan-Meier analysis showed no significant difference in the survival time among the three groups $(P=0.845)$. Conclusion: Mortality and morbidity increased in patients with LVD on hemodialysis compared with patients with normal EF.

Keywords: LVD, hemodialysis, mortality, hospitalization

\section{Introduction}

Left ventricular dysfunction (LVD) is characterized as a left ventricular ejection fraction (EF) below one-half of the systolic capacity of the left ventricle. Patients on hemodialysis have 10-30 times the risk of LVD than that of the general population. ${ }^{1-3}$ Recent literature has shown a correlation between cardiovascular disease (CVD) and chronic kidney disease (CKD), and how often they coexist. ${ }^{2}$ In patients with CKD, $74 \%$ have left ventricular hypertrophy at the beginning of dialysis, and it is considered to be the most common cardiac finding in those patients. ${ }^{4}$

Previous studies have shown that CVD contributes to most of the morbidity and mortality in patients receiving hemodialysis. Patients with CVD receiving hemodialy-
Correspondence: Marwan A Albeshri College of Medicine, King Abdulaziz University, North Abdullah AI Sulaiman Road, Jeddah 21589, Saudi Arabia Tel +966582903138

Email albeshrimarwan@gmail.com 
sis have up to 20 times higher mortality risk than the rest of the population. ${ }^{5-8}$ In patients with $\mathrm{CKD}$, the morbidity and mortality of CVD are abnormally high in all stages of CKD, with a predominance of $80 \%{ }^{6,9}$ Patients with LVD are more prone to comorbidities, such as high blood pressure, diabetes mellitus (DM), anemia, and a low body mass index. ${ }^{1-3}$ In dialysis patients, there are multiple risk factors that lead to CVD and contribute to the prognosis. Some of these risk factors include advanced age, systemic hypertension (HTN), DM, proteinuria, obesity, cigarette smoking, and the risk factors associated with renal impairment. DM and cigarette smoking have worsening effects on dialysis patients, with DM being shown to decrease the survival rate and increase the hospitalization rate among those patients. ${ }^{1-3,6,9}$

When comparing heart failure (HF) and low EF $(<40 \%)$ patients, those who were on dialysis were less likely to receive guideline-directed therapy when compared with those with no renal impairment. ${ }^{10}$ In LVD patients receiving hemodialysis, it is important to determine the impact of these risk factors on the prognosis, clinical outcome, survival rate, and prevalence of morbidity and mortality associated with this disease. Future guidelines and protocols dealing with this group of patients must be designed to improve the outcome and survival among those patients. For this research, our aim was to assess the clinical characteristics, comorbidities, hospitalization rate, and outcomes among hemodialysis patients with LVD compared with normal EF patients in the King Abdulaziz University Hospital (KAUH) Hemodialysis Unit in Jeddah, Saudi Arabia.

\section{Patients and methods Study design and participants}

We conducted this hospital-based retrospective cohort study at the Department of Medicine in the KAUH Hemodialysis Unit between November 2016 and September 2017 using the electronic-based patient records.

All patients $\geq 18$ years old, who started hemodialysis therapy at KAUH between January 2011 and December 2011, were identified using the hospital information system and medical records of the hemodialysis unit. The patients were then divided into three groups according to their EF results prior to starting hemodialysis as patients with $\mathrm{EF}<40 \%$, EF between $40 \%$ and $49 \%$, and $\mathrm{EF} \geq 50 \%$. Patients were then followed for 5 years by reviewing their hospital records to assess their outcomes, hospital admissions, and length of hospital stay (LOS).

\section{Variables and data measurements}

Using a standardized and pretested data extraction sheet, we collected data from the electronic hospital records and hemodialysis unit registry. The following data were extracted: age, gender, nationality, DM history, ischemic heart disease (IHD) (one or more vessels disease on coronary angiography), dyslipidemia, body mass index, height, weight, cause of end-stage renal disease, number of hospital admissions over the study period, mean LOS per visit, intensive care unit (ICU) stay, echocardiography results before starting dialysis therapy, mortality, and time to death in months. Left ventricular EF was assessed for all patients by certified, well-trained echocardiography technicians prior to the initiation of dialysis. The procedure and results were supervised, reported, and verified by a consultant in cardiology. The primary outcomes were mortality and time from the index visit to death. Secondary outcomes include mean LOS in each visit, ICU stay, and the total number of readmissions over the study period. Important demographic variables were identified a priori for inclusion as covariates (namely, age, sex, DM history, IHD, dyslipidemia, body mass index, and cause of end-stage renal disease).

\section{Statistical methods}

We used percentages to represent the categorical data. If the numerical data were normally distributed, we used the mean and SD, but we used the median and IQR if not. A chi-squared test was used when comparing the categorical variables. The Kruskal-Wallis test was used for the numerical variables. Correlations were done to examine the relation between numerical variables. Kaplan-Meier analysis was used to assess survival in all groups. To adjust for potential confounding variables, multiple logistic regression models were constructed. IBM SPSS Statistics for Windows, version 21.0 (IBM Corporation, Armonk, NY, USA) was used, and for all the statistical tests, a $P$-value of $<0.05$ was defined as the level of significance.

\section{Ethical considerations}

Ethical approval was obtained from the Department of Bioethics at KAUH. The requirement to obtain written informed consent from each patient was waived because this was an observational retrospective study. All patients' information were confidential, and data were analyzed anonymously.

\section{Results}

The analysis included 333 patients who were receiving hemodialysis. Of these, 257 patients had EFs $\geq 50 \%, 36$ had EFs of $40 \%-49 \%$, and 40 had EFs $<40 \%$. The age was significantly higher in patients with $\mathrm{EFs}<50 \%$ when compared with those 
with $\mathrm{EFs} \geq 50 \%$ ( $P=0.002)$. The proportion of males varies across the groups too (Table 1).

With regard to the comorbidities, $92.5 \%(\mathrm{~N}=37)$ and $86.1 \%(\mathrm{~N}=31)$ of patients with $\mathrm{EFs}<40 \%$ and $\mathrm{EFs}$ of $40 \%-49 \%$, respectively, had one or more comorbidities when compared with only $69 \%$ (N=177) of patients with EFs $\geq 50 \%(P<0.001)$. DM was prevalent in $70 \%$ of patients with EFs $<40 \%$ and $53 \%$ of patients with EFs of $40 \%-49 \%$ when compared with only $31 \%$ of those with EFs $\geq 50 \%(P<0.001)$. HTN was also more prevalent in patients with $\mathrm{EFs}<50 \%$; $88 \%(\mathrm{~N}=35)$ of patients with $\mathrm{EFs}<40 \%, 81 \%$ of those with EFs of $40 \%-49 \%$, and only $63 \%(\mathrm{~N}=163)$ of those with EFs $\geq 50 \%$ had HTN $(P=0.002$; Table 1$)$.

When comparing the mortality, $50 \%(\mathrm{~N}=20)$ of patients with $\mathrm{EFs}<40 \%$ and $44 \%$ ( $\mathrm{N}=16)$ with EFs of $40 \%-49 \%$ died when compared with $27 \%(\mathrm{~N}=68)$ of patients with $\mathrm{EFs} \geq 50 \%$ $(P=0.002)$ (Table 2). The median number of admissions in patients with $\mathrm{EFs}<40 \%$ was 2.5 (IQR $=7$ ), while in patients with EFs of $40 \%-49 \%$ it was 2 (IQR =6) compared with 2
(IQR =5) in patients with $\mathrm{EFs} \geq 50 \%(P=0.409)$. However, the average length of the stay between the three groups was significantly different $(P=0.007)$ (Table 2$)$. The number of ICU admissions showed a statistically significant difference between the three groups $(P=0.013)$ (Table 2).

When comparing echocardiographic parameters before starting hemodialysis among the three groups, left atrial size was significantly different among the three groups $(P<0.001)$, and it shows that the lower the EF in the classification, the larger the atrial size. The same findings are also observed in the left ventricular size, which was also significantly different among the three groups $(P<0.001)$. However, fractional shortening decreases with lower EF, and it is statistically different between the groups $(P<0.001)$ (Table 3$)$.

When correlating echocardiographic parameters with number of hospital admissions, EF was negatively correlated to number of admission (corr. coeff. $r=-0.005, P=0.928$ ). Left atrial size was positively correlated to number of admissions (corr. coeff. $r=0.053, P=0.414$ ). Left ventricular size

Table I General characteristics of patients according to their ejection fractions (EFs)

\begin{tabular}{|c|c|c|c|c|}
\hline Characteristics & $\begin{array}{l}E F<40 \% \\
(N=40)\end{array}$ & $\begin{array}{l}E F=40 \%-49 \% \\
(N=36)\end{array}$ & $\begin{array}{l}E F=50 \%-100 \% \\
(N=257)\end{array}$ & $P$-value \\
\hline \multicolumn{5}{|l|}{ Age } \\
\hline Mean [SD] & $61.08[16.1]$ & $60.9[13.9]$ & $53.43[16.8]$ & 0.002 \\
\hline Median [IQR] & $61[18]$ & $63[12]$ & $55[22]$ & \\
\hline \multicolumn{5}{|l|}{ Gender } \\
\hline Males & $26(65)$ & $24(69)$ & $126(49)$ & \multirow[t]{2}{*}{0.025} \\
\hline Females & $14(35)$ & II (3I) & $|3|(5 \mid)$ & \\
\hline Comorbidities, ${ }^{a} \mathrm{~N}(\%)$ & $37(92.5)$ & $31(86.1)$ & $177(68.9)$ & $<0.001$ \\
\hline Diabetes mellitus, N (\%) & $28(70)$ & $19(52.8)$ & $79(30.7)$ & $<0.001$ \\
\hline Hypertension, N (\%) & $35(87.5)$ & $29(80.6)$ & $163(63.4)$ & 0.002 \\
\hline Dyslipidemia, N (\%) & $5(12.5)$ & $\mathrm{I}(2.8)$ & $13(5.1)$ & 0.122 \\
\hline Ischemic heart disease, N (\%) & $16(40)$ & II (30.6) & $20(7.8)$ & $<0.001$ \\
\hline
\end{tabular}

Note: ${ }^{a}$ Comorbidities = hypertension, diabetes, ischemic heart disease, and dyslipidemia.

Table 2 Hospitalization rates and outcomes of patients according to their ejection fractions (EFs)

\begin{tabular}{|c|c|c|c|c|}
\hline Parameters & $\begin{array}{l}E F<40 \% \\
(N=40)\end{array}$ & $\begin{array}{l}E F=40 \%-49 \% \\
(N=36)\end{array}$ & $\begin{array}{l}E F=50 \%-100 \% \\
(N=257)\end{array}$ & $P$-value \\
\hline \multicolumn{5}{|c|}{ Number of admissions per 5 years } \\
\hline Median [SD] & $6.4[12.1]$ & $5.5[7.5]$ & $4.5[8.6]$ & 0.409 \\
\hline Median [IQR] & $2.5[7]$ & $2[6]$ & $2[5]$ & \\
\hline Minimum-maximum & $0-69$ & $0-27$ & $0-86$ & \\
\hline \multicolumn{5}{|c|}{ Average length of stay per admission (days) } \\
\hline Mean $[S D]$ & $5.05[5.5]$ & $13.8[20.3]$ & $6.8[13.1]$ & 0.007 \\
\hline Median [IQR] & $4[8]$ & $7[8]$ & $5[7]$ & \\
\hline Minimum-maximum & $0-30$ & $0-90$ & $0-180$ & \\
\hline $\begin{array}{l}\text { Intensive care unit } \\
\text { admission, } N(\%)\end{array}$ & $23(57.5)$ & $19(52.8)$ & $94(36.6)$ & 0.013 \\
\hline Death, N (\%) & $20(50.0)$ & $16(44.4)$ & $68(26.5)$ & 0.002 \\
\hline \multicolumn{5}{|l|}{ Time to death in months } \\
\hline Mean $[\mathrm{SD}]$ & $45.8[16]$ & $44[16.5]$ & $46[13]$ & 0.852 \\
\hline Median [IQR] & 49 & 51 & 50 & \\
\hline
\end{tabular}


Table 3 Echocardiographic parameters before starting hemodialysis among the three groups

\begin{tabular}{|c|c|c|c|c|}
\hline Parameters & $\begin{array}{l}E F<40 \% \\
(N=40)\end{array}$ & $\begin{array}{l}E F=40 \%-49 \% \\
(N=36)\end{array}$ & $\begin{array}{l}E F=50 \%-100 \% \\
(N=257)\end{array}$ & $P$-value \\
\hline \multicolumn{5}{|c|}{ Left atrium size } \\
\hline Mean [SD] & $4.5[0.8]$ & $4.3[0.8]$ & $3.9[0.7]$ & \multirow[t]{2}{*}{$<0.001$} \\
\hline Median & 5 & 4 & 4 & \\
\hline \multicolumn{5}{|c|}{ Left ventricle size } \\
\hline Mean [SD] & $4.8[0.8]$ & $4.0[0.6]$ & $3.6[0.6]$ & \multirow[t]{2}{*}{$<0.001$} \\
\hline Median & 5 & 4 & 3 & \\
\hline \multicolumn{5}{|c|}{ Fractional shortening } \\
\hline Mean [SD] & I6.7 [4.7] & $25.0[6.3]$ & $34.2[5.0]$ & \multirow[t]{2}{*}{$<0.001$} \\
\hline Median & 16.5 & 23 & 34 & \\
\hline
\end{tabular}

Abbreviation: EF, ejection fraction.

was also positively correlated to number of admissions (corr. coeff. $r=0.39, P=0.561$ ). Fractional shortening was positively correlated to number of admission (corr. coeff. $r=0.015, P=0.821$ ).

When correlating LOS with echocardiographic parameters, EF was negatively correlated to LOS (corr. coeff. $r=0.09 . P=0.141$ ). Left atrial size was positively correlated to LOS (corr. coeff. $r=0.182, P=0.004$ ). Left ventricular size was positively correlated to LOS (corr. coeff. $r=0.187$, $P=0.005)$. Fractional shortening was negatively correlated to LOS (corr. coeff. $r=-0.094, P=162$ ).

When comparing echocardiographic parameters before the initiation of hemodialysis and the mortality in our patients, EF before the initiation of hemodialysis was significantly lower in patients who died within the study period $(P<0.001)$. Fractional shortening was also significantly lower in patients who died. However, left atrial and ventricular sizes show no significant difference (Table 4).

In the logistic regression model for death, the age was significantly associated with mortality with an OR of 1.035 (95\% CI $=1.004-1.068)(P=0.027)$. DM had an OR of $2.270(95 \% \mathrm{CI}=0.795-6.482)(P=0.126)$, and ICU admission was significantly associated with increased mortality with an OR of 15.983 (95\% CI $=6.057-42.175)$ $(P<0.001$; Table 5).

Kaplan-Meier survival analysis was done to examine the survival among the three groups according to their EF. However, there was no statistically significant difference in the survival time in months among the three groups $(P=0.845)$ (Figure 1). Number of patients at risk over time is presented in Table 6.

The associations between the number of admissions and the other variables were examined using linear regression. The only significant factor was that of the left ventricular
Table 4 Characteristics of echocardiographic parameters before the initiation of hemodialysis according to the 5-year mortality

\begin{tabular}{|c|c|c|c|}
\hline Parameters & $\begin{array}{l}\text { Died } \\
(\mathrm{N}=104)\end{array}$ & $\begin{array}{l}\text { Survived } \\
(\mathrm{N}=\mathbf{2 2 9})\end{array}$ & $P$-value \\
\hline \multicolumn{4}{|c|}{ Ejection fraction } \\
\hline Mean [SD] & $50.7[15.7]$ & $57[12.3]$ & $<0.001$ \\
\hline Median & 55 & 60 & \\
\hline \multicolumn{4}{|l|}{ Left atrium size } \\
\hline Mean [SD] & $4[0.7]$ & $4[0.7]$ & \multirow[t]{2}{*}{0.394} \\
\hline Median & 4 & 4 & \\
\hline \multicolumn{4}{|c|}{ Left ventricle size } \\
\hline Mean [SD] & $3.8[0.9]$ & $3.6[0.8]$ & \multirow[t]{2}{*}{0.07} \\
\hline Median & 4 & 3 & \\
\hline \multicolumn{4}{|c|}{ Fractional shortening } \\
\hline Mean [SD] & $28.8[8.3]$ & $31[8.2]$ & \multirow[t]{2}{*}{0.047} \\
\hline Median & 31 & 32 & \\
\hline
\end{tabular}

Table 5 Logistic regression and OR results for mortality

\begin{tabular}{|c|c|c|}
\hline Variables & $P$-value & OR $(95 \% \mathrm{CI})$ \\
\hline Age (years) & 0.027 & $1.035(1.004-1.068)$ \\
\hline Gender & 0.401 & $\mathrm{I} .46 \mathrm{I}(0.603-3.536)$ \\
\hline Comorbidities & 0.898 & $1.148(0.138-9.546)$ \\
\hline Hypertension & 0.349 & $0.423(0.070-2.562)$ \\
\hline Ischemic heart disease & 0.923 & $0.946(0.307-2.917)$ \\
\hline Diabetes mellitus & 0.126 & $2.270(0.795-6.482)$ \\
\hline Dyslipidemia & 0.322 & $2.290(0.444-\mathrm{II} .80 \mathrm{I})$ \\
\hline Body mass index & 0.485 & $0.978(0.9|8-| .04 I)$ \\
\hline Number of admissions & 0.446 & $1.024(0.964-1.088)$ \\
\hline $\begin{array}{l}\text { Average length of } \\
\text { admission in days }\end{array}$ & 0.231 & $0.971(0.926-1.019)$ \\
\hline Intensive care unit & $<0.001$ & $15.983(6.057-42.175)$ \\
\hline Left atrium size & 0.797 & $1.089(0.569-2.084)$ \\
\hline Left ventricle size & 0.797 & $0.907(0.430-1.912)$ \\
\hline Fractional shortening & 0.228 & $1.073(0.957-1.204)$ \\
\hline Ejection fraction & 0.148 & $0.952(0.890-1.018)$ \\
\hline
\end{tabular}

size, which was negatively correlated $(\beta=-0.226)$ with the number of admissions ( $P=0.043$; Table 7$)$.

A linear regression was also estimated for average length of stay in each admission. The ICU admissions were posi- 


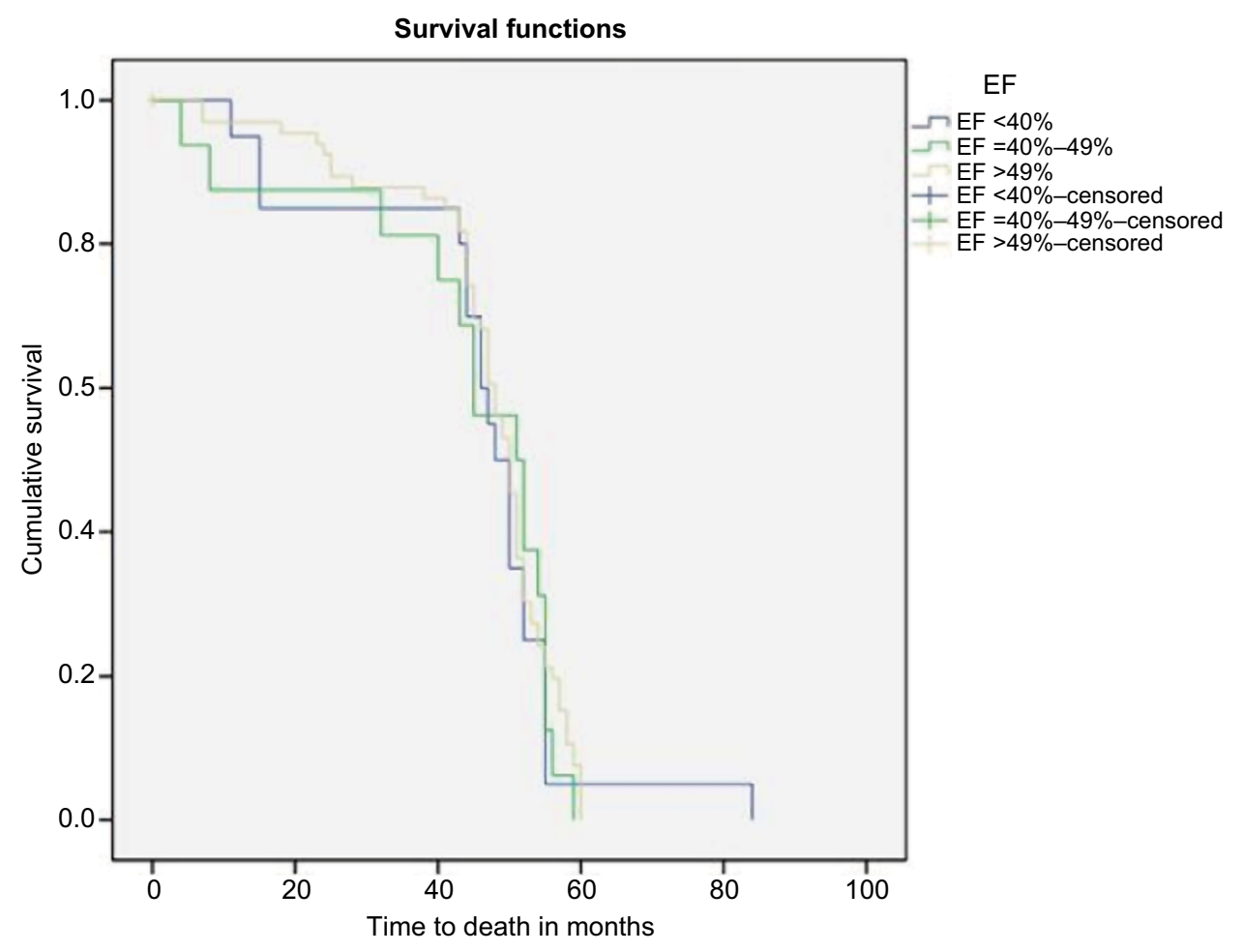

Figure I Kaplan-Meier survival analysis for patients according to their EF classification prior to enrolment in the study. Abbreviation: EF, ejection fraction.

tively correlated and associated with an increased length of stay $(\beta=0.332)(P<0.001)$. The other variables showed no significant correlations (Table 8).

\section{Discussion}

In our study, we compared the characteristics and hospitalization rates of dialysis patients based on their EFs. Those with EFs $>50 \%$ were considered to be the control group, and those with EFs $<40 \%$ and EFs of $40 \%-49 \%$ were considered to be the cases. Among the patients with EFs $<40 \%$ and EFs of $40 \%-49 \%$, there was an increased mortality rate when compared with the control group $(50 \%, 44.4 \%$, and $26.5 \%$, respectively). Our findings are supported by numerous previous studies, ${ }^{2,3}$ which showed that HF patients with low EFs associated with renal failure requiring dialysis had higher morbidity and mortality rates when compared with those without HF. ${ }^{11}$ This can be explained by the notion that with the increased duration of ERSD, changes may affect the cardiovascular system, like uremic pericarditis, and the serum calcium can affect both the blood vessels and the myocardium. This may eventually lead to the development of HF or a low EF, which some studies have shown to be risk factors for increasing the mortality rate among dialysis patients. ${ }^{2,12}$ However, the effect of EF on time to mortality in 5 years showed no significant difference between the three group, which may indicate that $\mathrm{EF}$ alone has minimal effect on time to mortality and other factors (demographics and comorbidities) must be taken under consideration when assessing time to mortality and improving the outcomes in those patients.

Our result also demonstrated that patients with EFs $<50 \%$ do indeed have a worse prognosis than those with $\mathrm{EFs} \geq 50 \%$. Although our present study did not show any significance in the multivariate analysis, this could be explained by the small sample size.

Only advanced age was found to be an independent risk factor for mortality in dialysis patients in our study. DM, HTN, dyslipidemia, and IHD were not significantly associated with mortality. It is worth noting that the lower the patients were in the classification, the higher the prevalence of the abovementioned comorbidities, which may reflect their poor cardiovascular profile. These findings are contradictory to many studies, ${ }^{3,5,8}$ which shows that DM and IHD, in addition to smoking and advanced age (a finding supporting one of our results), were associated with an increased mortality in those patients. The results of another previous study were consistent with ours with regard to age being a risk factor for mortality. ${ }^{13}$ A possible explanation for this diversity may be 
Table 6 Kaplan-Meier survival data

\begin{tabular}{|c|c|c|c|c|c|c|c|}
\hline \multirow{2}{*}{\multicolumn{2}{|c|}{ EF }} & \multirow{3}{*}{$\begin{array}{l}\text { Time } \\
\\
\text { II }\end{array}$} & \multirow{3}{*}{$\begin{array}{l}\begin{array}{l}\text { Status } \\
\text { estimate }\end{array} \\
\text { Died } \\
\end{array}$} & \multicolumn{2}{|c|}{$\begin{array}{l}\text { Cumulative proportion } \\
\text { surviving at the time }\end{array}$} & \multirow{3}{*}{$\begin{array}{l}\begin{array}{l}\text { No of } \\
\text { cumulative } \\
\text { events }\end{array} \\
\text { I } \\
\end{array}$} & \multirow{3}{*}{$\begin{array}{l}\begin{array}{l}\text { No of } \\
\text { remaining } \\
\text { cases }\end{array} \\
19 \\
\end{array}$} \\
\hline & & & & \multirow{2}{*}{$\begin{array}{l}\text { Estimate } \\
0.950 \\
\end{array}$} & \multirow{2}{*}{\begin{tabular}{|l|}
$\begin{array}{l}\text { Standard } \\
\text { error }\end{array}$ \\
0.049 \\
\end{tabular}} & & \\
\hline$E F<40 \%$ & 1 & & & & & & \\
\hline & 2 & 15 & Died & 0.0 & 0.0 & 2 & 18 \\
\hline & 3 & 15 & Died & 0.850 & 0.080 & 3 & 17 \\
\hline & 4 & 43 & Died & 0.800 & 0.089 & 4 & 16 \\
\hline & 5 & 44 & Died & 0.0 & 0.0 & 5 & 15 \\
\hline & 6 & 44 & Died & 0.700 & 0.102 & 6 & 14 \\
\hline & 7 & 46 & Died & 0.0 & 0.0 & 7 & 13 \\
\hline & 8 & 46 & Died & 0.600 & 0.110 & 8 & 12 \\
\hline & 9 & 47 & Died & 0.550 & 0.111 & 9 & 11 \\
\hline & 10 & 48 & Died & 0.500 & 0.112 & 10 & 10 \\
\hline & 11 & 50 & Died & 0.0 & 0.0 & 11 & 9 \\
\hline & 12 & 50 & Died & 0.0 & 0.0 & 12 & 8 \\
\hline & 13 & 50 & Died & 0.350 & 0.107 & 13 & 7 \\
\hline & 14 & 52 & Died & 0.0 & 0.0 & 14 & 6 \\
\hline & 15 & 52 & Died & 0.250 & 0.097 & 15 & 5 \\
\hline & 16 & 55 & Died & 0.0 & 0.0 & 16 & 4 \\
\hline & 17 & 55 & Died & 0.0 & 0.0 & 17 & 3 \\
\hline & 18 & 55 & Died & 0.0 & 0.0 & 18 & 2 \\
\hline & 19 & 55 & Died & 0.050 & 0.049 & 19 & $\mathrm{I}$ \\
\hline & 20 & 84 & Died & 0.000 & 0.000 & 20 & 0 \\
\hline \multirow[t]{16}{*}{$E F=40 \%-49 \%$} & $\mathrm{I}$ & 4 & Died & 0.938 & 0.061 & $\mathrm{I}$ & 15 \\
\hline & 2 & 8 & Died & 0.875 & 0.083 & 2 & 14 \\
\hline & 3 & 32 & Died & 0.813 & 0.098 & 3 & 13 \\
\hline & 4 & 40 & Died & 0.750 & 0.108 & 4 & 12 \\
\hline & 5 & 43 & Died & 0.688 & 0.116 & 5 & 11 \\
\hline & 6 & 45 & Died & 0.0 & 0.0 & 6 & 10 \\
\hline & 7 & 45 & Died & 0.563 & 0.124 & 7 & 9 \\
\hline & 8 & 51 & Died & 0.500 & 0.125 & 8 & 8 \\
\hline & 9 & 52 & Died & 0.0 & 0.0 & 9 & 7 \\
\hline & 10 & 52 & Died & 0.375 & 0.121 & 10 & 6 \\
\hline & 11 & 54 & Died & 0.313 & 0.116 & 11 & 5 \\
\hline & 12 & 55 & Died & 0.0 & 0.0 & 12 & 4 \\
\hline & 13 & 55 & Died & 0.0 & 0.0 & 13 & 3 \\
\hline & 14 & 55 & Died & 0.125 & 0.083 & 14 & 2 \\
\hline & 15 & 56 & Died & 0.063 & 0.061 & 15 & 1 \\
\hline & 16 & 59 & Died & 0.000 & 0.000 & 16 & 0 \\
\hline \multirow[t]{18}{*}{$E F>49 \%$} & 1 & 0 & 0.00 & 0.0 & 0.0 & 0 & 66 \\
\hline & 2 & 7 & Died & 0.0 & 0.0 & $I$ & 65 \\
\hline & 3 & 7 & Died & 0.970 & 0.021 & 2 & 64 \\
\hline & 4 & 18 & Died & 0.955 & 0.026 & 3 & 63 \\
\hline & 5 & 23 & Died & 0.939 & 0.029 & 4 & 62 \\
\hline & 6 & 24 & Died & 0.924 & 0.033 & 5 & 61 \\
\hline & 7 & 25 & Died & 0.0 & 0.0 & 6 & 60 \\
\hline & 8 & 25 & Died & 0.894 & 0.038 & 7 & 59 \\
\hline & 9 & 28 & Died & 0.879 & 0.040 & 8 & 58 \\
\hline & 10 & 38 & Died & 0.864 & 0.042 & 9 & 57 \\
\hline & 11 & $4 I$ & Died & 0.848 & 0.044 & 10 & 56 \\
\hline & 12 & 43 & Died & 0.0 & 0.0 & 11 & 55 \\
\hline & 13 & 43 & Died & 0.818 & 0.047 & 12 & 54 \\
\hline & 14 & 44 & Died & 0.0 & 0.0 & 13 & 53 \\
\hline & 15 & 44 & Died & 0.0 & 0.0 & 14 & 52 \\
\hline & 16 & 44 & Died & 0.0 & 0.0 & 15 & 51 \\
\hline & 17 & 44 & Died & 0.0 & 0.0 & 16 & 50 \\
\hline & 18 & 44 & Died & 0.742 & 0.054 & 17 & 49 \\
\hline
\end{tabular}


Table 6 (Continued)

\begin{tabular}{|c|c|c|c|c|c|c|c|}
\hline \multirow{2}{*}{\multicolumn{2}{|c|}{ EF }} & \multirow[t]{2}{*}{ Time } & \multirow[t]{2}{*}{$\begin{array}{l}\text { Status } \\
\text { estimate }\end{array}$} & \multicolumn{2}{|c|}{$\begin{array}{l}\text { Cumulative proportion } \\
\text { surviving at the time }\end{array}$} & \multirow{2}{*}{$\begin{array}{l}\text { No of } \\
\text { cumulative } \\
\text { events }\end{array}$} & \multirow{2}{*}{$\begin{array}{l}\text { No of } \\
\text { remaining } \\
\text { cases }\end{array}$} \\
\hline & & & & Estimate & $\begin{array}{l}\text { Standard } \\
\text { error }\end{array}$ & & \\
\hline & 20 & 45 & Died & 0.0 & 0.0 & 19 & 47 \\
\hline & 21 & 45 & Died & 0.697 & 0.057 & 20 & 46 \\
\hline & 22 & 46 & Died & 0.682 & 0.057 & 21 & 45 \\
\hline & 23 & 47 & Died & 0.0 & 0.0 & 22 & 44 \\
\hline & 24 & 47 & Died & 0.0 & 0.0 & 23 & 43 \\
\hline & 25 & 47 & Died & 0.0 & 0.0 & 24 & 42 \\
\hline & 26 & 47 & Died & 0.0 & 0.0 & 25 & 41 \\
\hline & 27 & 47 & Died & 0.606 & 0.060 & 26 & 40 \\
\hline & 28 & 48 & Died & 0.0 & 0.0 & 27 & 39 \\
\hline & 29 & 48 & Died & 0.0 & 0.0 & 28 & 38 \\
\hline & 30 & 48 & Died & $0.56 \mathrm{I}$ & 0.061 & 29 & 37 \\
\hline & 31 & 49 & Died & \begin{tabular}{|l|}
0.0 \\
\end{tabular} & 0.0 & 30 & 36 \\
\hline & 32 & 49 & Died & 0.530 & 0.061 & 31 & 35 \\
\hline & 33 & 50 & Died & 0.0 & 0.0 & 32 & 34 \\
\hline & 34 & 50 & Died & 0.0 & 0.0 & 33 & 33 \\
\hline & 35 & 50 & Died & 0.0 & 0.0 & 34 & 32 \\
\hline & 36 & 50 & Died & \begin{tabular}{|l|}
0.0 \\
\end{tabular} & 0.0 & 35 & 31 \\
\hline & 37 & 50 & Died & 0.455 & 0.061 & 36 & 30 \\
\hline & 38 & 51 & Died & 0.0 & 0.0 & 37 & 29 \\
\hline & 39 & 51 & Died & 0.0 & 0.0 & 38 & 28 \\
\hline & 40 & 51 & Died & 0.0 & 0.0 & 39 & 27 \\
\hline & 41 & 51 & Died & 0.0 & 0.0 & 40 & 26 \\
\hline & 42 & 51 & Died & 0.0 & 0.0 & 41 & 25 \\
\hline & 43 & 51 & Died & \begin{tabular}{|l|}
0.364 \\
\end{tabular} & 0.059 & 42 & 24 \\
\hline & 44 & 52 & Died & \begin{tabular}{|l|}
0.0 \\
\end{tabular} & 0.0 & 43 & 23 \\
\hline & 45 & 52 & Died & \begin{tabular}{|l|}
0.0 \\
\end{tabular} & 0.0 & 44 & 22 \\
\hline & 46 & 52 & Died & 0.0 & 0.0 & 45 & 21 \\
\hline & 47 & 52 & Died & 0.303 & 0.057 & 46 & 20 \\
\hline & 48 & 53 & Died & $\begin{array}{ll}0.0 \\
\end{array}$ & 0.0 & 47 & 19 \\
\hline & 49 & 53 & Died & 0.273 & 0.055 & 48 & 18 \\
\hline & 50 & 54 & Died & 0.0 & 0.0 & 49 & 17 \\
\hline & 51 & 54 & Died & \begin{tabular}{|l|}
0.242 \\
\end{tabular} & 0.053 & 50 & 16 \\
\hline & 52 & 55 & Died & \begin{tabular}{|l|}
0.0 \\
\end{tabular} & \begin{tabular}{|l|}
0.0 \\
\end{tabular} & 51 & 15 \\
\hline & 53 & 55 & Died & 0.212 & 0.050 & 52 & 14 \\
\hline & 54 & 56 & Died & \begin{tabular}{|l|l|}
0.197 \\
\end{tabular} & 0.049 & 53 & 13 \\
\hline & 55 & 57 & Died & \begin{tabular}{|l|}
0.0 \\
\end{tabular} & 0.0 & 54 & 12 \\
\hline & 56 & 57 & Died & 0.0 & 0.0 & 55 & 11 \\
\hline & 57 & 57 & Died & 0.152 & 0.044 & 56 & 10 \\
\hline & 58 & 58 & Died & \begin{tabular}{|l|}
0.0 \\
\end{tabular} & 0.0 & 57 & 9 \\
\hline & 59 & 58 & Died & 0.0 & 0.0 & 58 & 8 \\
\hline & 60 & 58 & Died & 0.106 & 0.038 & 59 & 7 \\
\hline & 61 & 59 & Died & \begin{tabular}{|l|}
0.0 \\
\end{tabular} & 0.0 & 60 & 6 \\
\hline & 62 & 59 & Died & \begin{tabular}{|l|}
0.076 \\
\end{tabular} & 0.033 & 61 & 5 \\
\hline & 63 & 60 & Died & \begin{tabular}{|l|}
0.0 \\
\end{tabular} & 0.0 & 62 & 4 \\
\hline & 64 & 60 & Died & 0.0 & 0.0 & 63 & 3 \\
\hline & 65 & 60 & Died & 0.0 & 0.0 & 64 & 2 \\
\hline & 66 & 60 & Died & 0.0 & 0.0 & 65 & 1 \\
\hline & 67 & 60 & Died & 0.000 & 0.000 & 66 & 0 \\
\hline
\end{tabular}

Abbreviation: EF, ejection fraction.

the sample size differences between our study and others, as their studies were larger. Additionally, we cannot exclude the potential variability present between different ethnicities and countries. ${ }^{14}$ These differences may shed some light on how different ethnicities have different risk factors, which may become an interesting topic to explore in the future.

One interesting finding in one study was that HTN was associated with lower mortality among patients on dialysis 
Table 7 Linear regression for number of admissions

\begin{tabular}{|c|c|c|c|c|c|c|c|}
\hline \multicolumn{8}{|l|}{ Coefficients } \\
\hline \multirow[t]{2}{*}{ Model } & \multicolumn{2}{|c|}{$\begin{array}{l}\text { Unstandardized } \\
\text { coefficients }\end{array}$} & \multirow{2}{*}{$\begin{array}{l}\text { Standardized } \\
\text { coefficients }\end{array}$} & \multirow[t]{2}{*}{$t$} & \multirow[t]{2}{*}{$P$-value } & \multicolumn{2}{|c|}{$95 \% \mathrm{Cl}$ for $\mathrm{B}$} \\
\hline & B & $\begin{array}{l}\text { Standard } \\
\text { error }\end{array}$ & & & & $\begin{array}{l}\text { Lower } \\
\text { bound }\end{array}$ & $\begin{array}{l}\text { Upper } \\
\text { bound }\end{array}$ \\
\hline Ejection fraction & -0.025 & 0.087 & -0.047 & -0.281 & 0.779 & -0.0197 & 0.148 \\
\hline Age & -0.019 & 0.035 & -0.048 & -0.534 & 0.594 & -0.088 & 0.051 \\
\hline Gender & -1.137 & 1.067 & -0.082 & -1.066 & 0.288 & -3.244 & 0.969 \\
\hline Comorbidities & -1.022 & 2.606 & -0.055 & -0.392 & 0.695 & -6.166 & 4.121 \\
\hline Hypertension & 0.347 & 2.313 & 0.021 & 0.150 & $0.88 I$ & -4.218 & 4.912 \\
\hline $\begin{array}{l}\text { Ischemic heart } \\
\text { disease }\end{array}$ & 1.107 & 1.484 & 0.064 & 0.746 & 0.457 & -1.821 & 4.035 \\
\hline Diabetes mellitus & 1.822 & 1.302 & 0.130 & 1.400 & 0.163 & -0.747 & 4.392 \\
\hline Dyslipidemia & 3.671 & 2.234 & 0.124 & 1.643 & 0.102 & -0.739 & 8.08 \\
\hline Body mass index & -0.081 & 0.076 & -0.081 & -1.058 & 0.291 & -0.231 & 0.070 \\
\hline $\begin{array}{l}\text { Average length of } \\
\text { admission in days }\end{array}$ & 0.018 & 0.069 & 0.020 & 0.257 & 0.798 & -0.118 & 0.154 \\
\hline Intensive care unit & 1.754 & 1.147 & 0.125 & 1.529 & 0.128 & -0.510 & 4.018 \\
\hline Left atrium size & 1.106 & 0.782 & 0.118 & 1.413 & 0.159 & -0.438 & 2.650 \\
\hline Left ventricle size & -1.909 & 0.937 & -0.226 & -2.037 & 0.043 & -3.759 & -0.059 \\
\hline $\begin{array}{l}\text { Fractional } \\
\text { shortening }\end{array}$ & 0.003 & 0.146 & 0.003 & 0.020 & 0.984 & -0.285 & 0.291 \\
\hline
\end{tabular}

Table 8 Linear regression for the average length of stay

\begin{tabular}{|c|c|c|c|c|c|c|c|}
\hline \multicolumn{8}{|l|}{ Coefficients } \\
\hline \multirow[t]{2}{*}{ Model } & \multicolumn{2}{|c|}{$\begin{array}{l}\text { Unstandardized } \\
\text { coefficients }\end{array}$} & \multirow{2}{*}{$\begin{array}{l}\text { Standardized } \\
\text { coefficients }\end{array}$} & \multirow[t]{2}{*}{$t$} & \multirow[t]{2}{*}{$P$-value } & \multicolumn{2}{|c|}{$95 \% \mathrm{Cl}$ for $\mathrm{B}$} \\
\hline & B & $\begin{array}{l}\text { Standard } \\
\text { error }\end{array}$ & & & & $\begin{array}{l}\text { Lower } \\
\text { bound }\end{array}$ & $\begin{array}{l}\text { Upper } \\
\text { bound }\end{array}$ \\
\hline Ejection fraction & 0.010 & 0.096 & 0.017 & 0.105 & 0.916 & -0.178 & 0.199 \\
\hline Age & 0.013 & 0.039 & 0.029 & 0.333 & 0.740 & -0.063 & 0.089 \\
\hline Gender & 0.944 & 1.167 & 0.062 & 0.809 & 0.420 & -1.359 & 3.248 \\
\hline Comorbidities & -0.947 & 2.847 & -0.046 & -0.333 & 0.740 & -6.565 & 4.671 \\
\hline Hypertension & 1.870 & 2.523 & 0.099 & $0.74 I$ & 0.460 & -3.109 & 6.848 \\
\hline Ischemic heart disease & 2.706 & 1.610 & 0.140 & 1.680 & 0.095 & -0.472 & 5.883 \\
\hline Diabetes mellitus & -0.697 & 1.429 & -0.045 & -0.488 & 0.626 & -3.517 & 2.123 \\
\hline Dyslipidemia & 0.562 & 2.458 & 0.017 & 0.229 & 0.819 & -4.290 & 5.414 \\
\hline Body mass index & -0.054 & 0.083 & -0.048 & -0.646 & 0.519 & -0.219 & 0.111 \\
\hline Intensive care unit & 5.003 & 1.204 & 0.321 & 4.157 & 0.000 & 2.628 & 7.379 \\
\hline Left atrium size & 0.782 & 0.857 & 0.075 & 0.912 & 0.363 & -0.910 & 2.474 \\
\hline Left ventricle size & 0.435 & 1.035 & 0.046 & 0.420 & 0.675 & -1.608 & 2.478 \\
\hline Fractional shortening & 0.169 & 0.159 & 0.177 & 1.066 & 0.288 & -0.144 & 0.483 \\
\hline Number of admissions & 0.021 & 0.082 & 0.019 & 0.257 & 0.798 & $-0.14 \mid$ & 0.184 \\
\hline
\end{tabular}

therapy. ${ }^{8}$ However, another study stated that HTN increased the mortality in dialysis patients. ${ }^{15}$ This disparity in the findings can be attributed to the differences in the populations studied, both in terms of the characteristics and number of centers included in other studies. According to the Hemodialysis (HEMO) Study, the most common cause of death in dialysis patients was of cardiac origin, amounting to $39.4 \%$ of the allcause death in the population under study. ${ }^{6}$ Another interesting finding in this study was the effect of using high flux dialyzer (the membrane is larger, to allow for the filtration of larger molecules) on decreasing cardiac cause of death. Another interesting finding regarded the effects of using a high-flux 
dialyzer (the membrane is larger to allow for the filtration of larger molecules) on decreasing the cardiac cause of death. ${ }^{16}$ In addition, it was found that with the use of high-flux dialyzer, there was a decrease in incidence of cardiac hospitalization. Unfortunately, in our study, the only type of dialyzer in our hospital is the low-flux type. As such, a comparison could not be made. This could shed some light on the importance of the type of dialyzer used in patients with LVD.

The number of ICU admissions was significantly higher in patients with EFs $<40 \%$ and EFs of $40 \%-49 \%$ than in the control group $(57.5 \%, 52.8 \%$, and $36.6 \%$, respectively). This can be explained by the fact that patients with low EFs are less likely to tolerate stressors, like fluid overload, uremic pericarditis, and electrolyte imbalances. The number of ICU admissions was also found to be an independent risk factor increasing both the mortality and the length of stay in our patients. This finding is supported by multiple studies, ${ }^{17,18}$ one of which shows that ERSD patients have a relative risk of death over the long term of 2.23 after admission to the ICU when compared with those ERSD patients without ICU admissions. In addition, that study found that age, $\mathrm{HF}$, and DM were risk factors for the 90-day mortality. ${ }^{18}$ One point that should be mentioned is that patients requiring dialysis may be more likely to be admitted to the ICU, either due to their vulnerability to infections ${ }^{19}$ or vascular access-associated complications. ${ }^{20}$ Thus, their condition may worsen, necessitating an admission to the ICU. An interesting finding was that the patients with EFs of $40 \%-49 \%$ had longer LOS in each admission when compared with those with EFs $<40 \%$, which is contradictory to the results of other studies. ${ }^{21-23}$ However, in the multivariate regression, the EF level did not show a significant association with the LOS. Further studies with larger sample sizes are needed to confirm the outcomes in this understudied group of hemodialysis patients.

The duration and frequency of dialysis treatments per week are highly associated with both the morbidity and mortality risks in those patients, as shown by previous research. Daily home dialysis and in-center dialysis have similar hospitalization risks, with the distinction that daily home hemodialysis has a lower risk of cardiovascular-related admissions in contrast to in-center dialysis. However, there is a higher risk of infection-related admissions. ${ }^{24} \mathrm{~A}$ systemic review and meta-analysis of randomized controlled trials was conducted revealing that every year between $10 \%$ and $20 \%$ of all dialysis patients die, with $\sim 45 \%$ of these deaths being due to underlying cardiovascular causes. ${ }^{25}$ One study conducted in 2001 showed that HF exhibited a higher increase in the risk of hospitalization in Caucasian patients by $16 \%$ when compared with African-American patients in which it was only $8 \% .^{14}$

This study did have some limitations; for example, it was conducted in only one center. In addition, it had a relatively small sample size when compared with other studies, and we lacked the specific causes of mortality in our patients. It may be interesting if the patients receiving hemodialysis were compared with those receiving peritoneal dialysis in terms of the EF under the same classification. Moreover, a study that encompasses most major centers in the region would exhibit a better representation of this population. However, our study is one of the first study to assess the mortality and outcomes in patients with LVD on hemodialysis in the region and in this specific population, which might further help in improving the guidelines and practice recommendations in patients with LVD on hemodialysis.

Our study demonstrated that a low EF can affect both the mortality and morbidity rates in patients receiving hemodialysis. In addition, advanced age and ICU admission were found to be independent risk factors for mortality in these hemodialysis patients.

\section{Conclusion}

Patients with LVD receiving hemodialysis have increased risks of mortality and morbidity when compared with those with normal EFs. The hospitalization rate showed no difference in those patients receiving hemodialysis with low and normal EFs. However, the LOS increased with a low EF. Advanced age and ICU admission were found to be independent risk factors for increased mortality and morbidity in these dialysis patients. A future prospective study with larger sample size is recommended to further study this group of patients and develop future guidelines and practices to improve the outcomes of patients with LVD and low EF.

\section{Acknowledgments}

We would like to thank Basma Salah Bamakhrama, Noor Jamal Baamer, Deema Siraj Abid, Eman Ghazi Darwish, and Wafaa Mohammed Alelyani for their help in data collection.

\section{Disclosure}

The authors report no conflicts of interest in this work.

\section{References}

1. Jassal SV, Trpeski L, Zhu N, Fenton S, Hemmelgarn B. Changes in survival among elderly patients initiating dialysis from 1990 to 1999. CMAJ. 2007;177(9):1033-1038.

2. Stack AG, Bloembergen WE. A cross-sectional study of the prevalence and clinical correlates of congestive heart failure among incident US dialysis patients. Am J Kidney Dis. 2001;38(5):992-1000. 
3. Harnett JD, Foley RN, Kent GM, Barre PE, Murray D, Parfrey PS. Congestive heart failure in dialysis patients: prevalence, incidence, prognosis and risk factors. Kidney Int. 1995;47(3):884-890.

4. Zolty R, Hynes PJ, Vittorio TJ. Severe left ventricular systolic dysfunction may reverse with renal transplantation: uremic cardiomyopathy and cardiorenal syndrome. Am J Transplant. 2008;8(11):2219-2224.

5. Levey AS, Beto JA, Coronado BE, et al. Controlling the epidemic of cardiovascular disease in chronic renal disease: what do we know? What do we need to learn? Where do we go from here? National Kidney Foundation Task Force on Cardiovascular Disease. Am J Kidney Dis. 1998;32(5):853-906.

6. Cheung AK, Sarnak MJ, Yan G, et al; HEMO Study Group. Cardiac diseases in maintenance hemodialysis patients: results of the HEMO Study. Kidney Int. 2004;65(6):2380-2389.

7. Sarnak MJ, Levey AS, Schoolwerth AC, et al; American Heart Association Councils on Kidney in Cardiovascular Disease, High Blood Pressure Research, Clinical Cardiology, and Epidemiology and Prevention. Kidney disease as a risk factor for development of cardiovascular disease: a statement from the American Heart Association Councils on Kidney in Cardiovascular Disease, High Blood Pressure Research. Hypertension. 2003;42(5):1050-1065.

8. Goodkin DA, Bragg-Gresham JL, Koenig KG, et al. Association of comorbid conditions and mortality in hemodialysis patients in Europe, Japan, and the United States: the Dialysis Outcomes and Practice Patterns Study (DOPPS). J Am Soc Nephrol. 2003;14(12):3270-3277.

9. Sarnak MJ. Cardiovascular complications in chronic kidney disease. Am J Kidney Dis. 2003;41(5 Suppl):11-17.

10. Pandey A, Golwala H, Devore AD, et al. Trends in the use of guidelinedirected therapies among dialysis patients hospitalized with systolic heart failure: findings from the American heart association get with the guidelines-heart failure program. JACC Heart Fail. 2016;4(8):649-661.

11. McCullough PA, Chan CT, Weinhandl ED, Burkart JM, Bakris GL. Intensive hemodialysis, left ventricular hypertrophy, and cardiovascular disease. Am J Kidney Dis. 2016;68(5S1):S5-S14.

12. Rostand SG, Sanders C, Kirk KA, Rutsky EA, Fraser RG. Myocardial calcification and cardiac dysfunction in chronic renal failure. Am JMed. 1988;85(5):651-657.

13. Lindner G, Doberer E, Vychytil A, et al. Prognosis in patients with congestive heart failure and subacute renal failure treated with hemodialysis. Wien Klin Wochenschr. 2009;121(11-12):391-397.
14. Collins AJ. Impact of congestive heart failure and other cardiac diseases on patient outcomes. Kidney Int Suppl. 2002;81(81):S3-S7.

15. Sharabas I, Siddiqi N. Cardiovascular disease risk profiles comparison among dialysis patients. Saudi J Kidney Dis Transpl. 2016;27(4):692-700.

16. Cheung AK, Levin NW, Greene T, et al. Effects of high-flux hemodialysis on clinical outcomes: results of the HEMO Study. J Am Soc Nephrol. 2003;14(12):3251-3263.

17. Clermont G, Acker CG, Angus DC, Sirio CA, Pinsky MR, Johnson JP. Renal failure in the ICU: comparison of the impact of acute renal failure and end-stage renal disease on ICU outcomes. Kidney Int. 2002;62(3):986-996.

18. Bell M, Granath F, Schön S, et al. End-stage renal disease patients on renal replacement therapy in the intensive care unit: short- and longterm outcome. Crit Care Med. 2008;36(10):2773-2778.

19. Kessler M, Hoen B, Mayeux D, Hestin D, Fontenaille C. Bacteremia in patients on chronic hemodialysis. A multicenter prospective survey. Nephron. 1993;64(1):95-100.

20. Sarnak MJ, Levey AS. Epidemiology, diagnosis, and management of cardiac disease in chronic renal disease. J Thromb Thrombolysis. 2000;10(2):169-180.

21. Carluccio E, Dini FL, Biagioli P, et al. The "Echo Heart Failure Score": an echocardiographic risk prediction score of mortality in systolic heart failure. Eur J Heart Fail. 2013;15(8):868-876.

22. Philbin EF, DiSalvo TG. Prediction of hospital readmission for heart failure: development of a simple risk score based on administrative data. J Am Coll Cardiol. 1999;33(6):1560-1566.

23. Philbin EF, Rocco TA, Lynch LJ, Rogers VA, Jenkins P. Predictors and determinants of hospital length of stay in congestive heart failure in ten community hospitals. J Heart Lung Transplant. 1997;16(5): 548-555.

24. McCullough PA, Chan CT, Weinhandl ED, Burkart JM, Bakris GL. Intensive hemodialysis, left ventricular hypertrophy, and cardiovascular disease. Am J Kidney Dis. 2016;68(5S1):S5-S14.

25. Heerspink HJ, Ninomiya T, Zoungas S, et al. Effect of lowering blood pressure on cardiovascular events and mortality in patients on dialysis: a systematic review and meta-analysis of randomised controlled trials. Lancet. 2009;373(9668):1009-1015.
International Journal of General Medicine

\section{Publish your work in this journal}

The International Journal of General Medicine is an international, peer-reviewed open-access journal that focuses on general and internal medicine, pathogenesis, epidemiology, diagnosis, monitoring and treatment protocols. The journal is characterized by the rapid reporting of reviews, original research and clinical studies across all disease areas.

\section{Dovepress}

The manuscript management system is completely online and includes a very quick and fair peer-review system, which is all easy to use. Visit http://www.dovepress.com/testimonials.php to read real quotes from published authors. 